\title{
FDTD calculation of the spontaneous emission coupling factor in optical microcavities
}

Jelena Vuckovic, Oskar J. Painter, Yong Xu, Amnon Yariv, Axel Scherer

Jelena Vuckovic, Oskar J. Painter, Yong Xu, Amnon Yariv, Axel Scherer, "FDTD calculation of the spontaneous emission coupling factor in optical microcavities," Proc. SPIE 3937, Micro- and Nano-photonic Materials and Devices, (17 April 2000); doi: 10.1117/12.382802 United States 


\title{
FDTD Calculation of the Spontaneous Emission Coupling Factor in Optical Microcavities
}

\author{
Jelena Vučković, Oskar Painter, Yong Xu, Amnon Yariv, Axel Scherer \\ Electrical Engineering and Applied Physics Departments \\ California Institute of Technology, 136-93 \\ Pasadena, CA 91125, U.S.A.
}

\begin{abstract}
We present the detailed analysis of the spontaneous emission coupling factor ( $\beta$ factor) of the microcavity based on a $2 \mathrm{D}$ photonic crystal in an optically thin dielectric slab. We investigate the maximum $\beta$ value that can be achieved with this microcavity and discuss its dependence on the quantum well position, as well as on the pumping area diameter. The analysis is performed using the general method for the $\beta$ factor calculation that we developed. The method is based on the classical model for atomic transitions in a semiconductor active medium. Finite difference time domain (FDTD) method is used to solve the electromagnetic fields of the system and calculate the total radiated energy, as well as the energy radiated into the mode of interest.
\end{abstract}

Keywords: $\beta$ factor, spontaneous emission, photonic crystals, microcavity, FDTD methods

\section{INTRODUCTION}

Over the past few years much scientific attention has been focused on the use of photonic crystals ${ }^{1}$ for building optical microcavities for spontaneous emission control ${ }^{2}$ and thresholdless lasing. ${ }^{3}$ One of the parameters which determines the quality of the microcavity is the spontaneous emission coupling factor ( $\beta$ factor). $\beta$ factor is defined as the ratio of the spontaneous emission rate into the mode of interest and the spontaneous emission rate into all modes. ${ }^{4}$ There are many analyses of this parameter in the literature, but they consider only simple laser geometries and often use many approximations. ${ }^{5-9}$ Our goal was to define a method for the calculation of this important parameter that would be flexible enough to incorporate highly complex geometries, including those of photonic crystals. We also wanted to investigate the possibility of using photonic crystals to build microcavities for low threshold lasers.

In section 2 we briefly describe our method for calculation of the spontaneous emission coupling factor. For the detailed description of the proposed method, the reader is referred to our previous work. ${ }^{10}$ In sections 3 and 4 we present the results of the $\beta$ factor calculation for the optical microcavity incorporating a two-dimensional photonic bandgap structure (2D PBG). We investigate the maximum $\beta$ value that can be obtained using this microcavity and analyze its dependence on the position of quantum well and the size of pumping area.

\section{DESCRIPTION OF THE PROPOSED METHOD}

Our starting point is the classical model for the $\beta$ factor calculation. ${ }^{5-7}$ Atomic transitions are modeled as classical oscillating electric dipoles with resonant frequencies equal to the atomic transition frequency $\omega_{p}$. Different dephasing mechanisms are taken into account through the dipole lifetime $\tau_{d}$, which corresponds to the homogeneous broadening of the spontaneous emission spectrum.

We solve the problem in a large box, which we call the computational domain and denote as $V_{C D}$. The first step in our calculation involves isolating the mode of interest in the optical cavity. Starting with an initial field distribution $\vec{E}(\vec{r}, t=0)$ and $\vec{H}(\vec{r}, t=0)$ we use the FDTD analysis to time evolve the electric and magnetic field in $V_{C D}$. Mur's absorbing boundary condition ${ }^{11}(\mathrm{ABC})$ are applied to the boundaries of the computational domain which allows the radiation to escape outside, without reflecting back into $V_{C D}$. During the time evolution, we store the field at a point of low symmetry in the microcavity. After applying a Fast Fourier Transform (FFT) to the resulting time

Further author information: Send correspondence to Jelena Vučković. E-mail: jela@caltech.edu 
series, we observe the resonant peaks in the spectrum corresponding to the modes of the structure. Then we filter the electromagnetic field for the mode of interest (what we term as the fundamental mode, whose electric field is denoted by $\vec{E}_{0}(\vec{r})$ ). The filtering is done by convolving the electromagnetic field in time with a bandpass filter centered at the resonant frequency of the fundamental mode and with an appropriate bandwidth. ${ }^{12,13}$ The filtered mode is then normalized in the following way:

$$
\iiint_{V_{C D}} \epsilon(\vec{r})\left|\vec{E}_{0}(\vec{r})\right|^{2} d^{3} \vec{r}=1
$$

At the same time, we calculate the quality factor $Q_{0}$ of the mode. Once we have solved for the mode of interest, we then proceed to calculate the electric and magnetic fields of the system excited by oscillating dipoles.

Let us assume we have $N$ dipoles, all at the same time in the microcavity, that are randomly positioned and randomly polarized in the active region $\left(\vec{r}_{p}^{(i)}\right.$ and $\hat{P}_{0}^{(i)}$ are the position and the polarization of the $i$-th dipole, respectively). We consider dipoles which have a single oscillation frequency $\omega_{p}$ and lifetime $\tau_{d}$, but random phases which are uniformly distributed in the range $[0,2 \pi)\left(\phi_{i}\right.$ is the initial phase of the $i$-th dipole). The Maxwell curl equations now have the following form:

$$
\begin{gathered}
\vec{\nabla} \times \vec{H}=\epsilon(\vec{r}) \frac{\partial \vec{E}}{\partial t}+\frac{\partial \vec{P}}{\partial t} \\
\vec{\nabla} \times \vec{E}=-\mu_{0} \frac{\partial \vec{H}}{\partial t}
\end{gathered}
$$

where $\vec{P}(\vec{r}, t)$ is given by the following expression:

$$
\vec{P}(\vec{r}, t)=\sum_{i=1}^{N} \hat{P}_{0}^{(i)} e^{j\left(\omega_{p} t+\phi_{i}\right)} e^{-\frac{t}{r_{d}}} \delta\left(\vec{r}-\vec{r}_{p}^{(i)}\right)
$$

We discretize the equations (2)-(4) with the initial conditions $\vec{E}\left(\vec{r}, t=0^{-}\right)=0$ and $\vec{H}\left(\vec{r}, t=0^{-}\right)=0$. Mur's ABCs are again applied to the boundaries of the computational domain $V_{C D}$.

Let us choose a volume $V$ (with outer surface $S$ ) to be a subset of $V_{C D}$ containing all the dipoles and enclosing the microcavity. If we evolve the fields for a long enough time $t^{\prime}\left(t^{\prime} \gg \tau_{d}\right)$, such that the energy of the electromagnetic field which remains within $V$ at $t=t^{\prime}$ is negligible, we can approximate the total energy radiated into all modes, $W_{\sum \infty}$, as:

$$
W_{\sum \infty} \approx \int_{0}^{t^{\prime}} P_{r a d}(\tau) d \tau
$$

where $P_{\text {rad }}(\tau)$ represents the power radiated out of the cavity at instant of time $\tau$ and is calculated as:

$$
P_{\text {rad }}(\tau)=\frac{1}{2} \iint_{S}\left(\vec{E}(\vec{r}, \tau) \times \vec{H}^{*}(\vec{r}, \tau)\right) d \vec{S}
$$

The transverse electric field (which is the radiation field) can be expressed as the superposition of the complete set of transverse orthonormal modes of the closed, lossless cavity $\left\{\vec{E}_{m}(\vec{r})\right\}:{ }^{14-16}$

$$
\vec{E}_{T}(\vec{r}, t)=\sum_{n} \vec{E}_{n}(\vec{r}) g_{n}(t)
$$

The orthonormality condition (with a position dependent dielectric constant $\epsilon(\vec{r})$ ) is defined as: ${ }^{15}$

$$
\iint_{V_{C D}} \epsilon(\vec{r}) \vec{E}_{m}(\vec{r}) \cdot \vec{E}_{n}^{*}(\vec{r}) d^{3} \vec{r}=\delta_{m n},
$$


where $\delta_{m n}$ is the Kronecker's delta. The transversality condition is given by: ${ }^{15}$

$$
\vec{\nabla} \cdot\left(\epsilon(\vec{r}) \vec{E}_{m}(\vec{r})\right)=0
$$

Each of these modes satisfies the following wave equation:

$$
\vec{\nabla} \times\left[\vec{\nabla} \times \vec{E}_{m}(\vec{r})\right]=\mu_{0} \epsilon(\vec{r}) \omega_{m}^{2} \vec{E}_{m}(\vec{r}),
$$

where $\omega_{m}$ is the frequency of a mode. Starting from the orthonormality and transversality conditions, we can easily solve for $g_{m}(t)$ (we assume that $g_{m}(0)=0$ for all $m$ ):

$$
\begin{aligned}
g_{m}(t) & =B_{m}\left(e^{j \omega_{p} t} e^{-\frac{t}{\tau_{d}}}-e^{j \omega_{m} t} e^{-\frac{\omega_{m} t}{2 Q_{m}}}\right) \\
B_{m} & =\frac{G^{(m)}}{\omega_{m}^{2}-\omega_{p}^{2}+\frac{1}{\tau_{d}^{2}}-\frac{\omega_{m}}{\tau_{d} Q_{m}}-j \omega_{p}\left(\frac{2}{\tau_{d}}-\frac{\omega_{m}}{Q_{m}}\right)} \\
G^{(m)} & =\left[-\omega_{p}^{2}+\frac{1}{\tau_{d}^{2}}-j \frac{2 \omega_{p}}{\tau_{d}}\right] \sum_{i=1}^{N} \hat{P}_{0}^{(i)} \cdot \vec{E}_{m}^{*}\left(\vec{r}_{p}^{(i)}\right) e^{j \phi_{i}},
\end{aligned}
$$

where $Q_{m}$ denotes the quality factor of the $m$-th mode.

Let us label the fundamental mode by index 0 . Then the energy radiated into the fundamental mode at time $t \rightarrow \infty$ can be calculated as: ${ }^{6}$

$$
W_{0 \infty}=\frac{1}{2} \int_{0}^{\infty} 2 \kappa_{0} d t \iiint_{V_{C D}} \epsilon(\vec{r}) \vec{E}_{0}(\vec{r}, t) \cdot \vec{E}_{0}^{*}(\vec{r}, t) d^{3} \vec{r}
$$

where

$$
\vec{E}_{0}(\vec{r}, t)=\vec{E}_{0}(\vec{r}) g_{0}(t)
$$

and the field decay rate $\kappa_{0}$ of the fundamental mode is defined as:

$$
\kappa_{0}=\frac{\omega_{0}}{2 Q_{0}}
$$

Then the total energy radiated into the fundamental mode is:

$$
W_{0 \infty}=\kappa_{0}\left|B_{0}\right|^{2}\left[\frac{\tau_{d}}{2}+\frac{Q_{0}}{\omega_{0}}-\frac{2\left(\frac{1}{\tau_{d}}+\frac{\omega_{0}}{2 Q_{0}}\right)}{\left(\omega_{p}-\omega_{0}\right)^{2}+\left(\frac{1}{\tau_{d}}+\frac{\omega_{0}}{2 Q_{0}}\right)^{2}}\right]
$$

The $\beta$ factor of the fundamental mode is equal to the ratio of the total energy radiated into that mode (given by expression (18)) and the total energy radiated into all modes (given by expression (5)). ${ }^{5-7}$

The inhomogenous broadening is taken into account by using an approximate transition spectrum which has a Lorentzian shape, with the central frequency $\omega_{s}$ and FWHM equal to $\Delta \omega_{s}$ :

$$
\begin{gathered}
F\left(w_{p}\right)=\frac{\frac{\Delta \omega_{s}}{2 \pi}}{\left(\omega_{p}-\omega_{s}\right)^{2}+\left(\frac{\Delta \omega_{s}}{2}\right)^{2}} \\
\int_{-\infty}^{+\infty} F\left(\omega_{p}\right) d \omega_{p}=1
\end{gathered}
$$


$F\left(w_{p}\right)$ represents the density of dipoles (electronic states) at the frequency $w_{p}$. We average the result over different dipole resonant frequencies:

$$
\beta=\frac{\sum_{\omega_{p}} F\left(\omega_{p}\right) W_{0 \infty}\left(\omega_{p}\right)}{\sum_{\omega_{p}} F\left(\omega_{p}\right) W_{\sum \infty}\left(\omega_{p}\right)}
$$

The value of the $\beta$ factor when only homogeneous broadening is taken into account and dipoles have the frequency $\omega_{p}$ is denoted by $\beta_{H}$ and given by:

$$
\beta_{H}=\frac{W_{0 \infty}\left(\omega_{p}\right)}{W_{\sum \infty}\left(\omega_{p}\right)}
$$

\section{3. $\beta$ FACTOR OF THE MICROCAVITY BASED ON 2D PBG IN AN OPTICALLY THIN DIELECTRIC SLAB}

Optical microcavities can be constructed by introducing defects into photonic crystals. ${ }^{1}$ These microcavities support high- $Q$, localized modes (defect modes), which have their resonant frequencies within the bandgap of the photonic crystal.

The microcavity that we analyze is an optically thin dielectric slab patterned with a 2D array of holes. A defect is made by omitting a central hole in the 2D array of air holes. A 2D PBG is used to laterally confine the defect mode and to suppress the lateral radiation. Total internal reflection at the air-membrane boundary is used for vertical confinement of the mode. The important parameters of the structure are the distance between centers of adjacent air holes $a$, the radius of an air hole $r$, the number of periods of holes around the defect $n_{p e r}$, the thickness of the membrane $d$, and the refractive index of the membrane $n_{\text {slab }}$. The parameters of the analyzed structure are: $\frac{r}{a}=0.3$, $\frac{d}{a}=0.4, n_{\text {slab }}=3.4$ and $n_{\text {per }}=3$. The detailed analysis of localized defect modes in this structure was presented in reference 13. In Figure 1 we show the top view of a microfabricated defect cavity in InGaAsP, designed for $1.55 \mu \mathrm{m}$ emission. ${ }^{17}$

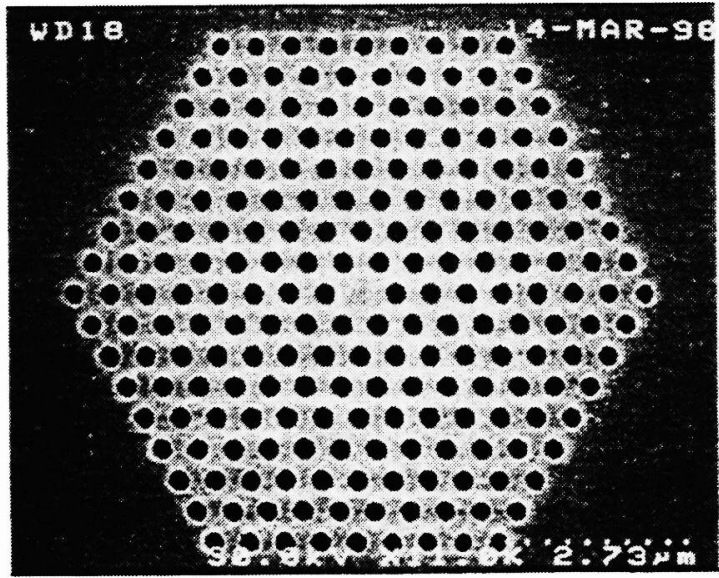

(a)

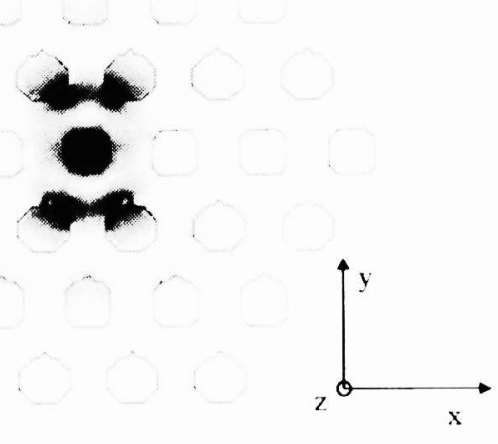

(b)

Figure 1. (a) Top view of a microfabricated $2 \mathrm{D}$ hexagonal array of holes with a single central hole missing. The inter-hole spacing is $a=500 \mathrm{~nm}$ and the radius of the holes is approximately $150 \mathrm{~nm}$; (b) $2 \mathrm{D}$ slice through the middle of the slab showing the y dipole mode electric field amplitude.

The defect modes are a set of doubly degenerate dipole modes, which we term the $x$-dipole and $y$-dipole mode. ${ }^{13}$ We consider the $y$-dipole shown in Figure 1 mode to be the fundamental mode. The normalized frequency of the 
fundamental mode is $\frac{a}{\lambda_{0}}=0.32$ and its quality factor is $Q_{0}=129$. If we assume that in reality $\lambda_{0}=1.55 \mu m$, this corresponds to $d=198 \mathrm{~nm}, a=495 \mathrm{~nm}, r=165 \mathrm{~nm}$. The bandgap of the infinite 2D photonic crystal of this thickness extends from $\frac{a}{\lambda}=0.29$ to $\frac{a}{\lambda}=0.38$.

The active layer $(\mathrm{QW})$ is centered in the middle of the dielectric membrane. We assume that the QW couples most strongly to TE modes, so we only analyze dipoles with the polarization in the plane of the QW. Dipoles appear uniformly throughout the area of the defect. The lifetime of dipoles is assumed to be $\tau_{d}=0.1 p s$. In order to account for the inhomogeneous broadening, we approximate the emission spectrum from the $\mathrm{QW}$ by a Lorentzian, centered at $\lambda_{s}$ and with FWHM denoted by $\Delta \lambda_{s}$. Using the averaging technique described in section 2, we calculate the value of the $\beta$ factor for different values of the inhomogeneous broadening and the central frequency of the spectrum matched to the frequency of the fundamental mode. The results are given in Figure 2.

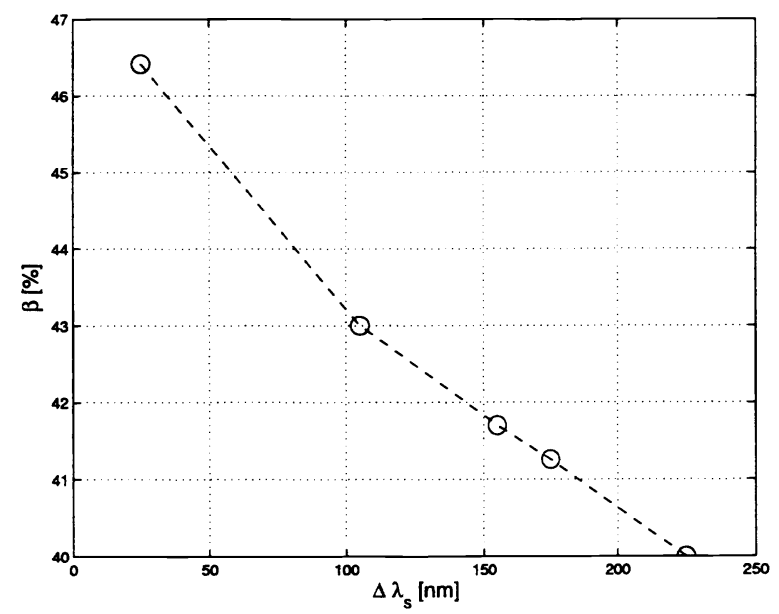

Figure 2. $\beta$ factor calculation for the y-dipole mode of the microcavity based on 2D PBG in an optically thin dielectric slab; parameters of the cavity are described in text. On $\mathrm{x}$ axis we plot the FWHM of the emission spectrum. It is assumed that the emission is centered around $\lambda_{0}=1.55 \mu m$.

We calculated the total energy radiated by a single electric dipole positioned in the center of the defect and oriented in the $x$ or $y$ direction for a range of dipole wavelengths $\lambda_{p}$. The dipole lifetime was assumed to be $0.1 p s$. The result is shown in Figure 3. It can be observed that the total energies radiated by $x$ and $y$ oriented electric dipoles are approximately the same. This is explained by the fact that the fundamental mode is a set of doubly degenerate dipole modes and the $x$ oriented electric dipole strongly couples to y-dipole mode, while the $y$ oriented electric dipole strongly couples to $\mathrm{x}$-dipole mode. As expected, the total radiated energies reach their maximum when dipole's wavelength coincides with the wavelength of the fundamental mode. When dipole's wavelength is offset from the wavelength of the fundamental mode in either direction, the total radiated energy drops, because we move within the bandgap and there is no other mode that the electric dipole can transfer its energy to. The drop in the total energy is gradual, due to the fact that the dipole emission spectrum is $25 \mathrm{~nm}$ wide and at small distances from $\lambda_{0}$ tails of the emission spectrum still couple to the fundamental mode. Within the bandgap and off $\lambda_{0}$ we would have the inhibited spontaneous emission. A very interesting observation is that the total energy radiated by a dipole starts to increase slowly for $a / \lambda_{p}>0.35$. This is due to coupling of dipole emission to conduction band modes (bottom of the conduction band is at $a / \lambda_{p}=0.38$ ). One would expect the similar increase in the total radiated energy at lower frequencies, due to the coupling of dipoles to the valence band modes (valence band top is positioned at $a / \lambda_{p}=0.29$ ). However, such increase is not observed in our calculations. This is explained by the fact that valence (dielectric) band modes have their electric field energy concentrated in the high dielectric constant regions in the unperturbed photonic crystal. On the other hand, we position the electric dipole in the center of the defect, which corresponds to the air (hole) region in the unperturbed photonic crystal. This means that the electric field intensity of the valence band modes is small at electric dipole position and their coupling is weak. Therefore, there is no increase in the energy radiated by an electric dipole positioned in the center of a defect, due to the coupling to 
valence band modes. This also explains the high $\beta$ values achievable for large FWHM of the emission spectrum, as shown in Figure 2, when the pumping area is small and limited to the defect. Therefore, in order to obtain a large $\beta$ value in this type of the microcavity for large FWHM of the emission spectrum, it is desirable to have a defect mode frequency closer to the valence band top than to the conduction band bottom and to pump only the defect area. Unfortunately, the later is very difficult to achieve in practice.

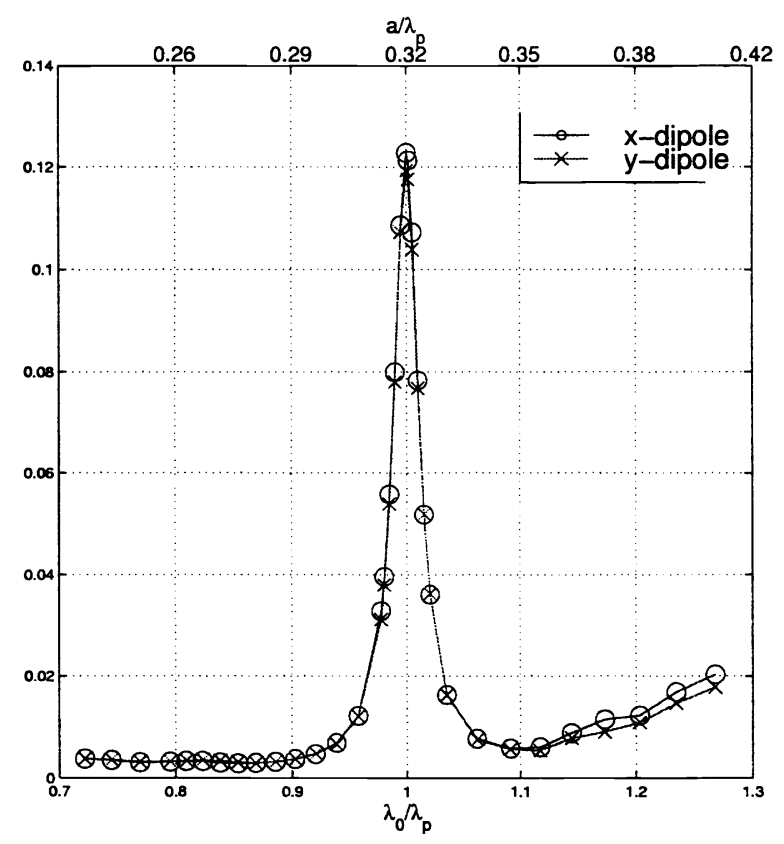

Figure 3. Total energy (in arbitrary units) radiated by a single electric dipole oriented in the $x$ or $y$ direction and positioned in the center of the defect. The microcavity is based on a 2D PBG in an optically thin dielectric slab. On the bottom $x$ axis we show the ratio of the wavelength of the fundamental mode $\lambda_{0}$ and the dipole's wavelength $\lambda_{p}$. On the top $x$ axis we represent the ratio of the interhole spacing $a$ and the dipole's wavelength $\lambda_{p}$.

\section{4. $\beta$ FACTOR OF THE MICROCAVITY WITH GEOMETRY FOR SPLITTING OF THE DIPOLE MODE DEGENERACY}

The $\beta$ factor can be almost doubled using the degeneracy splitting of the dipole modes. Degeneracy splitting can be accomplished by lowering the defect symmetry relative to that of the hexagonal lattice (as an example, by increasing the radius of the two nearest neighbor holes along the $\mathrm{x}$-axis). ${ }^{13}$ In the previously analyzed PBG membrane we increase the radii of the two nearest neighbor holes to $0.5 a$ and move them $0.2 a$ simultaneously toward the center defect in order to maintain the rib size in the $\mathrm{x}$ direction. We analyze the $\beta$ factor of the $\mathrm{y}$-dipole mode (shown in Figure 4), whose parameters are $Q=188$ and $\frac{a}{\lambda_{0}}=0.34$. The applied discretization was 15 units per interhole spacing $a$. Numbers on $\mathrm{x}$ and $\mathrm{y}$ axes of the field plots denote discretization units. In this microcavity, the $\mathrm{x}$-dipole mode is pushed completely outside the band-gap, as can be seen from the field spectrum shown in Figure 5 .

Using the method described in previous sections, we calculate the $\beta$ factor for this microcavity when only homogeneous broadening is taken into account. The homogeneous broadening is $25 \mathrm{~nm}$, corresponding to dipole's lifetime of $0.1 p s$ at $\lambda_{0}=1.55 \mu \mathrm{m}$. The emitting region is again assumed to be a single TE $\mathrm{QW}$, positioned in the middle of the membrane. In order to decrease the amount of computation, we average $\beta$ only over a single $x$ and $y$ oriented dipole positioned in the center of the defect. This approximation is good enough if the pumping area is small and limited to the defect. We test a range of dipole wavelengths $\lambda_{p}$. The result is shown in Figure 6 . When dipole wavelength matches the wavelength of the fundamental mode and the FWHM of the emission spectrum is equal to 
$25 \mathrm{~nm}, \beta$ factor as high as $85 \%$ can be achieved.
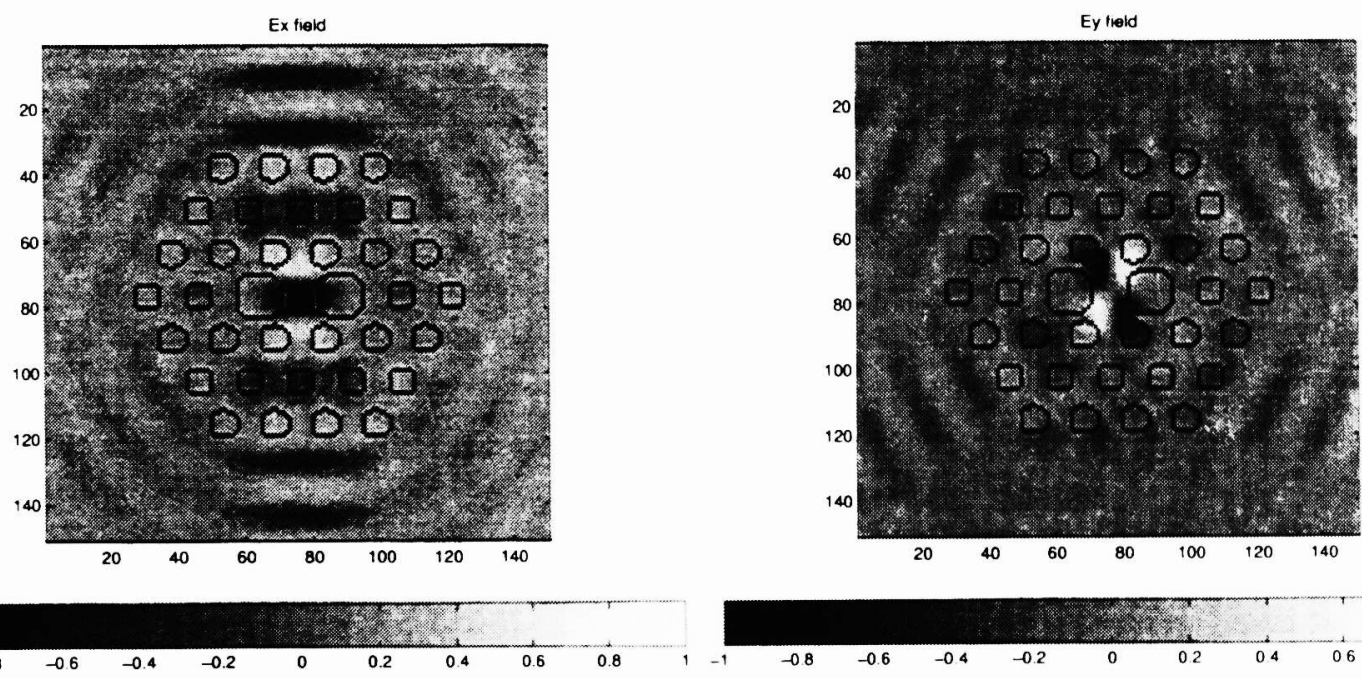

(a)

(b)

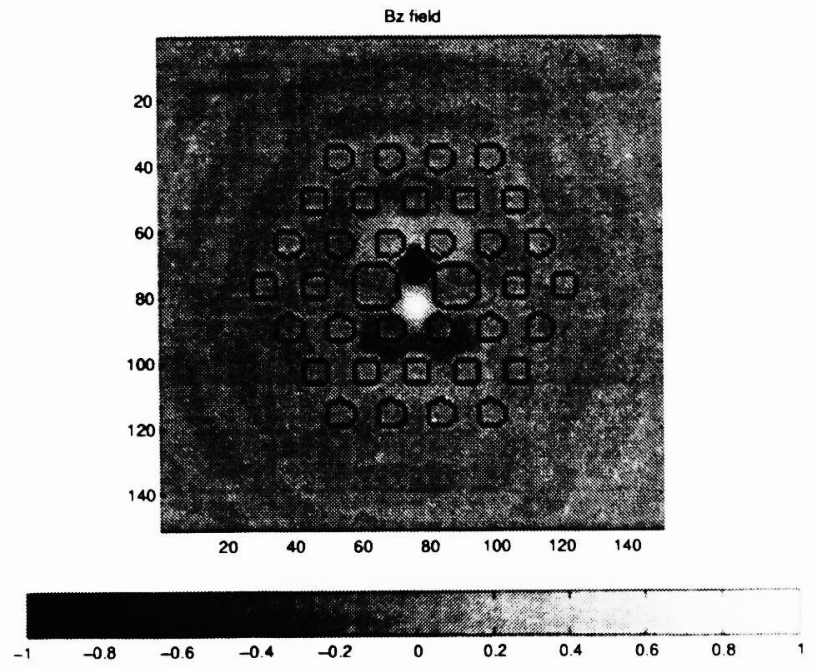

(c)

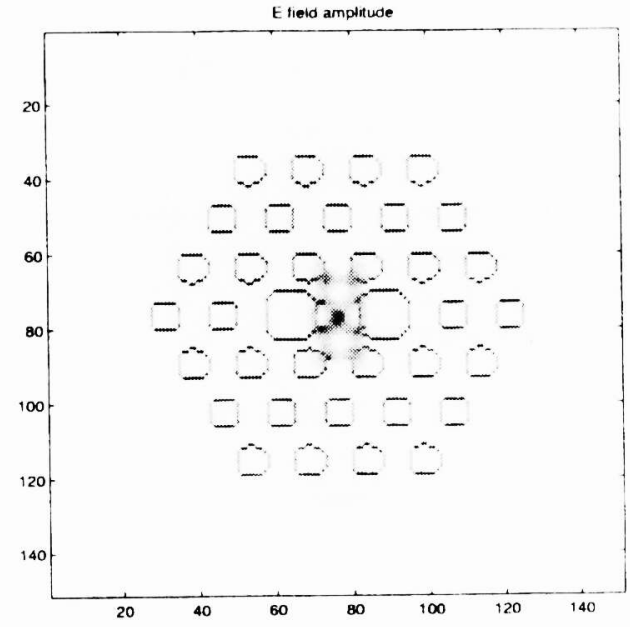

(d)

Figure 4. Cavity geometry for splitting of the dipole mode degeneracy and 2D slice through the middle of the slab showing the y dipole mode: (a) $x$ component of the electric field; (b) $y$ component of the electric field; (c) $z$ component of the magnetic field; (d) amplitude of the electric field (dark areas denote the high intensity regions at the amplitude plot ). Field components $E_{z}, B_{x}$ and $B_{y}$ are negligible (TE mode).

Once again, we calculated the total energy radiated by a single electric dipole positioned in the center of the defect and oriented in the $x$ or $y$ direction for a range of dipole wavelengths $\lambda_{p}$. The dipole lifetime was assumed to be $0.1 \mathrm{ps}$. The result is shown in Figure 7 . The total energy radiated by the $y$ oriented electric dipole is negligible within the bandgap. This can be explained by the fact that the $y$ oriented electric dipole couples strongly to the $\mathrm{x}$-dipole mode, which is pushed out of the bandgap with this cavity geometry. On the other hand, the total energy 
radiated by the $x$ oriented electric dipole is still very strong within the bandgap, since it couples to the y-dipole mode which is located in the bandgap. A rise in the total radiated energy for both dipole orientations is still observed at high frequencies and the explanation from the previous section still holds.

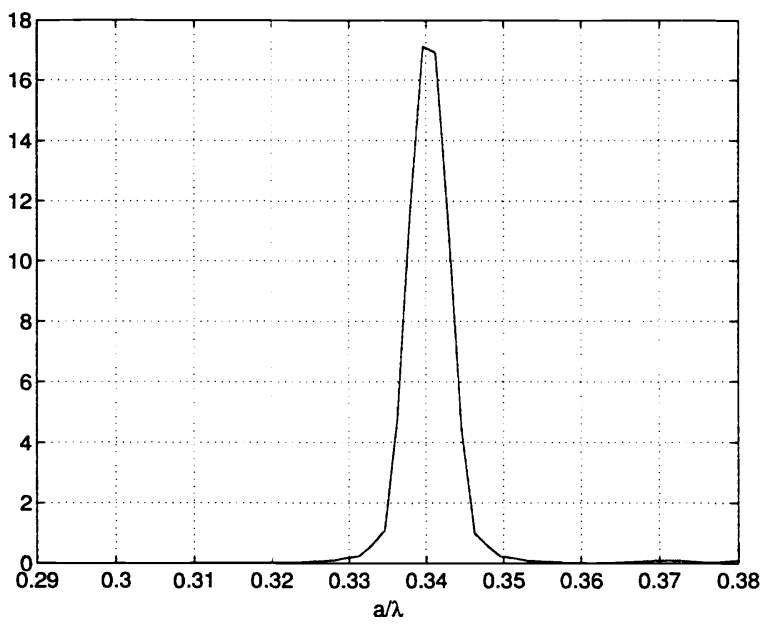

Figure 5. Field spectrum for the microcavity designed for degeneracy splitting of the dipole mode. The radii of the two nearest neighbor holes are increased from $0.3 a$ to $0.5 a$ and they are moved $0.2 a$ towards the center. The shown peak corresponds to the y-dipole mode, while the $\mathrm{x}$-dipole mode is completely pushed out of the bandgap. The bandgap extends from $a / \lambda=0.29$ to $a / \lambda=0.38$.

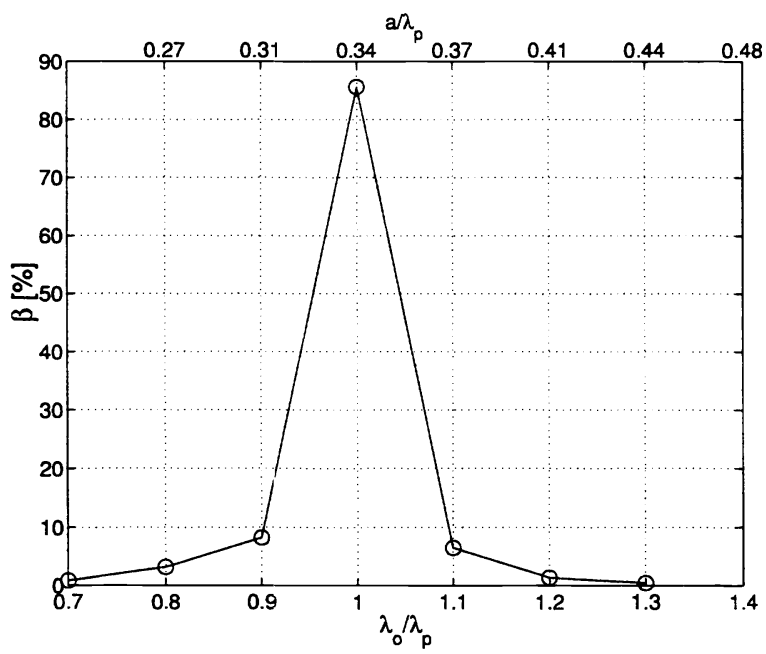

Figure 6. $\beta$ factor dependence on the wavelength of the dipole excitation $\lambda_{p}$ for the microcavity designed for degeneracy spliting of the dipole mode. Only the homogeneous broadening is taken into account.

In all previous calculations, it was assumed that the active region is a single quantum well positioned in the center of the membrane. We wanted to test the influence of the $\mathrm{QW}$ offset from the middle of the membrane to the $\beta$ factor. We analyzed $\beta$ of the same structure at $\lambda_{p}=\lambda_{0}$, but for QW positioned $\Delta z$ above the middle of the membrane. The result is shown in Figure 8. The drop in $\beta$ due to the $\mathrm{QW}$ offset is small. This is due to the fact that the electric field intensity of the $y$-dipole mode doesn't change very strongly along $\mathrm{z}$ direction within a membrane. ${ }^{13}$ 


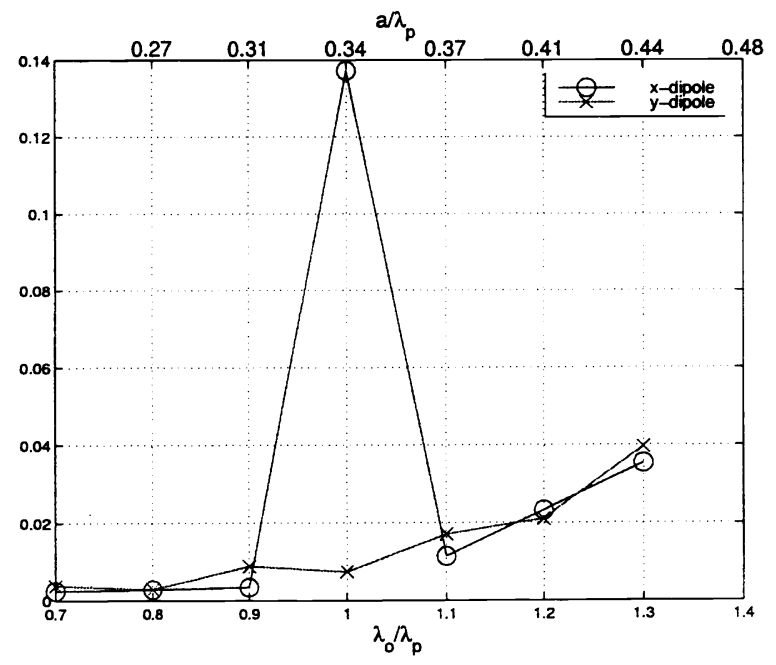

Figure 7. Total energy (in arbitrary units) radiated by a single electric dipole oriented in the $x$ or $y$ direction and positioned in the center of the defect. The microcavity geometry is designed for degeneracy splitting of the dipole mode and parameters are given in text. On the bottom $x$ axis we show the ratio of the fundamental mode wavelength $\lambda_{0}$ and the dipole wavelength $\lambda_{p}$. On the top $x$ axis we represent the ratio of the interhole spacing $a$ and the dipole's wavelength $\lambda_{p}$.

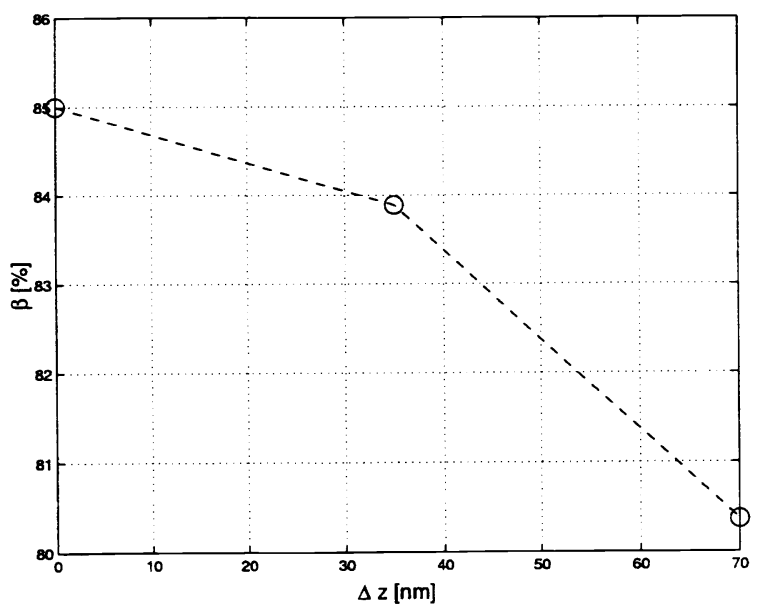

Figure 8. $\beta$ factor dependence on the position of the quantum well. The microcavity geometry is designed for degeneracy splitting of the dipole mode and parameters are given in text. On the $x$ axis we show the vertical distance of the quantum well from the middle of the membrane. Dipole wavelength is matched to the mode wavelength and only the homogeneous broadening is taken into account.

\section{CONCLUSION}

We proposed a new method for the calculation of the spontaneous emission coupling factor using the finite-difference time domain method. We calculated $\beta$ of the microcavity based on the 2D PBG structure. We investigated the maximum $\beta$ value that could be obtained using this geometry and analyzed its dependence on the position of quantum well and the size of the pumping region. 


\section{ACKNOWLEDGMENTS}

The authors would like to thank Marko Lončar for many helpful suggestions.

This work was supported by the NSF under contract ECS-9632937 and by DARPA under the contract N00014-96$1-1295$.

\section{REFERENCES}

1. J. D. Joannopoulos, R. D. Meade, and J. N. Winn, Photonic Crystals, Princeton University Press, Princeton, New Jersey, 1995.

2. E. Yablonovitch, "Inhibited Spontaneous Emission in Solid-State Physics and Electronics," Physical Review Letters 58, pp. 2059-2062, May 1987.

3. H. Yokoyama, "Physics and Device Applications of Optical Microcavities," Science 256, pp. 66-70, Apr. 1992.

4. G. Björk and Y. Yamamoto, "Analysis of Semiconductor Microcavity Lasers Using Rate Equations," IEEE Journal of Quantum Electronics 27, pp. 2386-2396, Nov. 1991.

5. Y. Suematsu and K. Furuya, "Theoretical Spontaneous Emission Factor of Injection Lasers," The Transactions of the IECE of Japan E60, pp. 467-472, Sept. 1977.

6. T. Baba, T. Hamano, F. Koyama, and K. Iga, "Spontaneous Emission Factor of a Microcavity DBR SurfaceEmitting Laser," IEEE Journal of Quantum Electronics 27, pp. 1347-1358, June 1991.

7. T. Baba, T. Hamano, F. Koyama, and K. Iga, "Spontaneous Emission Factor of a Microcavity DBR SurfaceEmitting Laser (II) - Effects of Electron Quantum Confinements," IEEE Journal of Quantum Electronics 28, pp. 1310-1319, May 1992.

8. M. Chin, D. Y. Chu, and S. Ho, "Estimation of the Spontaneous Emission Factor for Microdisk Lasers via the Approximation of Whispering Gallery Modes," Journal of Applied Physics 75, pp. 3302-3307, Apr. 1994.

9. D. Y. Chu and S. Ho, "Spontaneous Emission from Excitons in Cylindrical Dielectric Waveguides and the Spontaneous-Emission Factor of Microcavity Ring Lasers," Journal of the Optical Society of America B 10, pp. 381-390, Feb. 1993.

10. J. Vučković, O. Painter, and A. Scherer, "Finite-Difference Time Domain Calculation of The Spontaneous Emission Coupling Factor in Optical Microcavities," IEEE Journal of Quantum Electronics 35, pp. 1168-1175, Aug. 1999.

11. G. Mur, "Absorbing Boundary Conditions for the Finite-Difference Approximation of the Time-Domain Electromagnetic-Field Equations," IEEE Transactions on Electromagnetic Compatibility EMC-23, pp. 377382, Nov. 1981.

12. D.H.Choi and W.J.R.Hoefer, "The Finite-Difference-Time-Domain Method and its Application to Eigenvalue Problems," IEEE Transactions on Microwave Theory and Techniques 34, pp. 1464-1469, Dec. 1986.

13. O. Painter, J. Vučković, and A. Scherer, "Defect Modes of a Two-Dimensional Photonic Crystal in an Optically Thin Dielectric Slab," Journal of the Optical Society of America B 16, pp. 275-285, Feb. 1999.

14. E. A. Hinds, "Perturbative Cavity Quantum Electrodynamics," Cavity QED, edited by Paul. R. Berman, Academic Press, pp. 1-55, 1994.

15. R. J. Glauber and M. Lewenstein, "Quantum optics of dielectric media," Physical Review A 43(1), pp. 467-491, 1991.

16. Y. Xu, J. Vučković, R. K. Lee, O. J. Painter, A. Scherer, and A. Yariv, "Finite-difference time-domain calculation of spontaneous emission lifetime in a microcavity," Journal of the optical society of America B 16(3), pp. 465474, 1999.

17. O. Painter, R. Lee, A. Yariv, A. Scherer, and J. O'Brien, "Photonic bandgap membrane microresonator," Integrated Photonics Research 4, pp. 221-223, 1998. 